\title{
Benchmark Results on the Linearized Equations of Motion of an Uncontrolled Bicycle
}

\author{
A. L. Schwab \\ Laboratory for Engineering Mechanics \\ Delft University of Technology \\ Mekelweg 2, NL-2628 CD Delft, The Netherlands \\ Phone: +31-15-2782701, Fax: +31-15-2782150 \\ Email: a.I.schwab@wbmt.tudelft.nl
}

\author{
J. P. Meijaard ${ }^{1}$ and J. M. Papadopoulos ${ }^{2}$ \\ ${ }^{1}$ School of MMMEM \\ The University of Nottingham \\ University Park, Nottingham NG7 2RD, United Kingdom \\ ${ }^{2}$ The Paper Converting Machine Company \\ Green Bay, Wisconsin, USA
}

Keywords: Vehicle Dynamics, Non-Holonomic Constraints, Dynamic Stability.

\begin{abstract}
In this paper we present the linearized equations of motion for a bicycle as a benchmark. The results obtained by pencil-and-paper and two programs are compared. The bicycle model we consider here consists of four rigid bodies, viz. a rear frame, a front frame being the front fork and handlebar assembly, a rear wheel and a front wheel, which are connected by revolute joints. The contact between the knife-edge wheels and the flat level surface is modelled by holonomic constraints in the normal direction and by non-holonomic constraints in the longitudinal and lateral direction. The rider is rigidly attached to the rear frame with hands free from the handlebar. This system has three degrees of freedom, the roll, the steer, and the forward speed. For the benchmark we consider the linearized equations for small perturbations of the upright steady forward motion. The entries of the matrices of these equations form the basis for comparison. Three different kinds of methods to obtain the results are compared: pencil-and-paper, the numeric multibody dynamics program SPACAR, and the symbolic software system AutoSim. Because the results of the three methods are the same within the machine round-off error, we assume that the results are correct and can be used as a bicycle dynamics benchmark.
\end{abstract}

\section{Introduction}

A variety of simple vehicles can be statically unstable yet dynamically stable, for example a skateboard plus rigidly attached rider, a tricycle with raked steering axis, or a bicycle/motorcycle. Of these the bicycle is the most interesting, yet the hardest to analyse correctly. As a result the literature contains a great many flawed equations, and widespread qualitative explanations of uncontrolled self-stability are inconsistent with careful analyses.

It is the purpose of this paper to present exhaustively confirmed linearized equations of motion suitable for research or application. A second aim is to present a high-precision benchmark for the linearized governing equations for a single clearly defined bicycle travelling at a range of speeds. Alternative equation formulations, or even non-linear simulation of a small perturbation, can therefore be checked with confidence.

The study of bicycle and motorcycle dynamics has attracted attention from mechanical engineers such as Rankine [14], Sommerfeld and Klein [10], Timoshenko [24], Den Hartog [3], Neřmark and Fufaev [12], Kane [9] and many others also out of the engineering discipline. Investigations have ranged from purely ad hoc analyses to full non-linear computer simulations.

The first publication of the full non-linear and also the linearized equations of motion for an upright uncontrolled bicycle was by Whipple [28] in 1899. His linearization was found to be correct except for typographical errors. They agree with Döhring [4], with Neřmark and Fufaev [12] (after correcting errors due to an incorrect potential energy), with Sharp [22] (with a minor algebraic correction for the case of knife-edge wheels), with the Ph.D. thesis by Weir [26], with Weir and Zellner [27] (after correcting a sign error), and with the equations as derived by Hand [5], and simplified by Papadopoulos [13]. Moreover, Hand's work also gives a detailed literature review. Correct equations for more or less simplified models are presented by Carvallo [2], Klein and Sommerfeld [10], Timoshenko and Young [24], and Kane [9]. 


\section{PREPRINT}

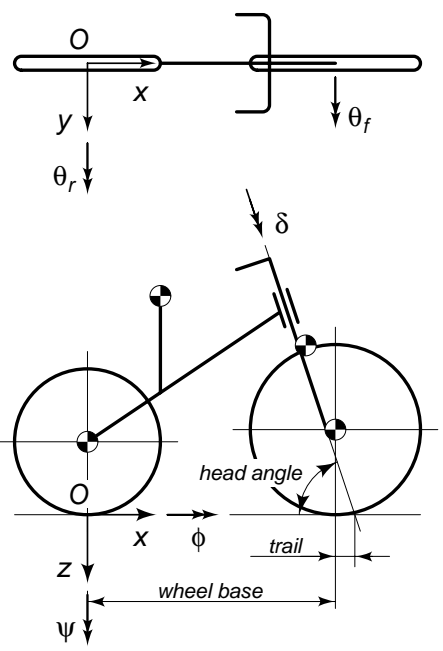

Figure 1. Bicycle model together with the coordinate system, the degrees of freedom, and the parameters.

Other authors' equations disagree with these and with each other's, or are so complex in presentation that detailed comparison was not practical.

\section{Bicycle Model}

The mechanical model of the bicycle consists of four rigid bodies, viz. the rear frame with the rider rigidly attached to it, the front frame being the front fork and handle bar assembly and the two knife-edge wheels. These bodies are interconnected by revolute hinges at the steering head between the rear frame and the front frame and at the two wheel hubs. In the reference configuration, all bodies are symmetric relative to the bicycle midplane. The contact between the stiff non-slipping wheels and the flat level surface is modelled by holonomic constraints in the normal direction and by nonholonomic constraints in the longitudinal and lateral direction. There is no friction, apart from the idealized friction between the non-slipping wheels and the surface, nor propulsion and no rider control, the so-called hands free coasting operation. These assumptions make the model energyconserving. In the reference position, the global Cartesian coordinate system is located at the rear-wheel contact point $O$, where the $\mathrm{x}$-axis points in the longitudinal direction of the bicycle and the z-axis is directed downwards. Figure 1 shows the directions of the axes, where the terminology used mainly follows the SAE recommended practice as described in the report SAE-J607e [15], last revised in 1976.

The mechanical model of the bicycle has three degrees of freedom: the roll angle $\phi$ of the rear frame, the steering angle $\delta$, and the rotation $\theta_{r}$ of the rear wheel with respect to the rear frame. The angles are defined as follows. The orientation of the rear frame with respect to the global reference frame $O-x y z$ is given by a sequence of three angular

\begin{tabular}{|c|c|c|c|}
\hline Parameter & Symbol & \multicolumn{2}{|l|}{ Value } \\
\hline Wheel base & $\bar{w}$ & \multicolumn{2}{|l|}{$1.02 \mathrm{~m}$} \\
\hline Trail & $t$ & \multicolumn{2}{|l|}{$0.08 \mathrm{~m}$} \\
\hline Head angle & $\alpha$ & \multicolumn{2}{|l|}{$\arctan (3)$} \\
\hline Gravity & $g$ & \multicolumn{2}{|l|}{$9.81 \mathrm{~N} / \mathrm{kg}$} \\
\hline Forward speed & $v$ & \multicolumn{2}{|l|}{ variable $\mathrm{m} / \mathrm{s}$} \\
\hline \multicolumn{4}{|l|}{ Rear wheel } \\
\hline Radius & $R_{r w}$ & \multirow{3}{*}{\multicolumn{2}{|c|}{$\begin{array}{l}0.3 \mathrm{~m} \\
2 \mathrm{~kg} \\
(0.06,0.12,0.06) \mathrm{kgm}^{2}\end{array}$}} \\
\hline Mass & & & \\
\hline Mass moments of inertia & $\left(A_{x x}, A_{y y}, A_{z z}\right)$ & & \\
\hline \multicolumn{4}{|l|}{ Rear frame } \\
\hline Position centre of mass & $\left(x_{r f}, y_{r f}, z_{r f}\right)$ & \multirow{3}{*}{\multicolumn{2}{|c|}{$\begin{array}{l}(0.3,0,-0.9) \mathrm{m} \\
85 \mathrm{~kg} \\
{\left[\begin{array}{ccc}9.2 & 0 & 2.4 \\
& 11 & 0 \\
& & 2.8\end{array}\right] \mathrm{kgm}^{2}}\end{array}$}} \\
\hline Mass & $m_{r f}$ & & \\
\hline Mass moments of inertia & {$\left[\begin{array}{ccc}B_{x x} & 0 & B_{x z} \\
& B_{y y} & 0 \\
s y m & & B_{y z}\end{array}\right]$} & & \\
\hline \multicolumn{4}{|l|}{ Front frame } \\
\hline $\begin{array}{l}\text { Position centre of mass } \\
\text { Mass }\end{array}$ & $\begin{array}{c}\left(x_{f f}, y_{f f}, z_{f f}\right) \\
m_{f f}\end{array}$ & \multirow{2}{*}{$\begin{array}{l}(0.9,0,-0.7) \mathrm{m} \\
4 \mathrm{~kg} \\
{\left[\begin{array}{ccc}0.0546 & 0 & -0.0162 \\
& 0.06 & 0 \\
& & 0.0114\end{array}\right]}\end{array}$} & \\
\hline Mass moments of inertia & {$\left[\begin{array}{ccc}C_{x x} & 0 & C_{x z} \\
& C_{y y} & 0 \\
\text { sym. } & & C_{z z}\end{array}\right]$} & & $\mathrm{kgm}^{2}$ \\
\hline \multicolumn{4}{|l|}{ Front wheel } \\
\hline $\begin{array}{l}\text { Radius } \\
\text { Mass } \\
\text { Mass moments of inertia }\end{array}$ & $\begin{array}{c}R_{f w} \\
m_{f w} \\
\left(D_{x x}, D_{y y}, D_{z z}\right) \\
\end{array}$ & \multicolumn{2}{|l|}{$\begin{array}{l}0.35 \mathrm{~m} \\
3 \mathrm{~kg} \\
(0.14,0.28,0.14) \mathrm{kgm}^{2}\end{array}$} \\
\hline
\end{tabular}

Table 1. Parameters for the benchmark bicycle from Figure 1.

rotations: a yaw rotation, $\psi$, about the $z$-axis, a roll rotation, $\phi$, about the rotated $x$-axis, and a pitch rotation, $\theta$, about the rotated $y$-axis. These rotations are materialized and depicted in Figure 4 by three hinges in series, (1), (2), and (3), mounted at the rear hub. The steering angle $\delta$ is the rotation of the front frame with respect to the rear frame about the steering axis. Due to the non-holonomic constraints there are four extra kinematic coordinates which describe, together with the degrees of freedom, the configuration of the system [18]. The four kinematic coordinates are taken here as the Cartesian coordinates $x$ and $y$ of the rear-wheel contact point, the yaw angle $\psi$ of the rear frame, and the rotation $\theta_{f}$ of the front wheel with respect to the front frame.

The dimensions and mechanical properties of the benchmark model are presented in Table 1 . The system is symmetric about the vertical longitudinal plane and the wheels are rotationally symmetric about their axles. The mass moments of inertia are given at the centre of mass of the individual bodies and along the global $x y z$-axes.

\section{Linearized Equations of Motion}

This section gives an algorithmic interpretation of the linearized equations of motion for the bicycle model under study as derived by Papadopoulos [13]. The equations of motion are obtained by pencil-and-paper using D'Alembert's principle and linear and angular momentum balances. They are expressed in terms of small changes in the degrees of free- 


\section{PREPRINT}

dom $\phi$, the rear frame roll angle, and $\delta$, the steering angle, from the upright straight ahead configuration $\phi_{0}=0, \delta_{0}=0$, at a forward speed of $v=-\dot{\theta}_{r} R_{r w}$.

Let us consider the bicycle from Figure 1 and Table 1. The subscripts used are: $r w$ for the rear wheel, $r f$ for the rear frame, $f f$ for the front frame, $f w$ for the front wheel, $t$ for the total system, $f$ for the front assembly which is the front frame plus the front wheel, $x, y$, and $z$ are the directions along the global $x y z$-axes, and $\lambda$ is the direction of the steering axis pointing downward. Then the algorithm is as follows. For the system as a whole, calculate the total mass and the corresponding centre of mass with respect to the origin $O$ as

$$
\begin{aligned}
m_{t} & =m_{r w}+m_{r f}+m_{f f}+m_{f w}, \\
x_{t} & =\left(x_{r f} m_{r f}+x_{f f} m_{f f}+w m_{f w}\right) / m_{t}, \\
z_{t} & =\left(-R_{r w} m_{r w}+z_{r f} m_{r f}+z_{f f} m_{f f}-R_{f w} m_{f w}\right) / m_{t} .
\end{aligned}
$$

For the system as a whole, calculate the relevant mass moments and products of inertia at the origin $O$ along the global axes as

$$
\begin{aligned}
T_{x x}= & A_{x x}+B_{x x}+C_{x x}+D_{x x}+m_{r w} R_{r w}^{2}+m_{r f} z_{r f}^{2}+ \\
& m_{f f} z_{f f}^{2}+m_{f w} R_{f w}^{2}, \\
T_{x z}= & B_{x z}+C_{x z}-m_{r f} x_{r f} z_{r f}-m_{f f} x_{f f} z_{f f}+m_{f w} w R_{f w}, \\
T_{z z}= & A_{z z}+B_{z z}+C_{z z}+D_{z z}+m_{r f} x_{r f}^{2}+m_{f f} x_{f f}^{2}+m_{f w} w^{2}
\end{aligned}
$$

Now determine the same properties for the front assembly, being the front frame and the front wheel, as

$$
\begin{aligned}
m_{f} & =m_{f f}+m_{f w}, \\
x_{f} & =\left(x_{f f} m_{f f}+w m_{f w}\right) / m_{f}, \\
z_{f} & =\left(z_{f f} m_{f f}-R_{f w} m_{f w}\right) / m_{f},
\end{aligned}
$$

and calculate the relevant mass moments and products of inertia for the front assembly at the centre of mass of the front assembly along the global axes as

$$
\begin{aligned}
F_{x x}= & C_{x x}+D_{x x}+m_{f f}\left(z_{f f}-z_{f}\right)^{2}+m_{f w}\left(R_{f w}+z_{f}\right)^{2} \\
F_{x z}= & C_{x z}-m_{f f}\left(x_{f f}-x_{f}\right)\left(z_{f f}-z_{f}\right)+ \\
& m_{f w}\left(w-x_{f}\right)\left(R_{f w}+z_{f}\right) \\
F_{z z}= & C_{z z}+D_{z z}+m_{f f}\left(x_{f f}-x_{f}\right)^{2}+m_{f w}\left(w-x_{f}\right)^{2} .
\end{aligned}
$$

Let $\lambda$ be the angle of the steering axis $\boldsymbol{\lambda}=(\sin (\lambda), 0, \cos (\lambda))^{T}$ with the global $z$-axis in the vertical plane,

$$
\lambda=\pi / 2-\alpha
$$

Calculate the perpendicular distance that the centre of mass of the front assembly is ahead of the steering axis,

$$
u=\left(x_{f}-w-t\right) \cos (\lambda)-z_{f} \sin (\lambda) .
$$

Calculate for the front assembly the relevant mass moments and products of inertia along the steering axis and the global axes at points where they intersect as

$$
\begin{aligned}
F_{\lambda \lambda}= & m_{f} u^{2}+F_{x x} \sin (\lambda)^{2}+2 F_{x z} \sin (\lambda) \cos (\lambda)+ \\
& F_{z z} \cos (\lambda)^{2} \\
F_{\lambda x}= & -m_{f} u z_{f}+F_{x x} \sin (\lambda)+F_{x z} \cos (\lambda) \\
F_{\lambda z}= & m_{f} u x_{f}+F_{x z} \sin (\lambda)+F_{z z} \cos (\lambda) .
\end{aligned}
$$

Define the ratio of the mechanical trail (i.e. the perpendicular distance that the front wheel contact point is behind the steering axis) to the wheelbase as

$$
f=t \cos (\lambda) / w
$$

Calculate for the rear and the front wheel the angular momentum along the $y$-axis divided by the forward speed, together with their sum as

$$
\begin{aligned}
S_{r} & =A_{y y} / R_{r w}, \\
S_{f} & =D_{y y} / R_{f w}, \\
S_{t} & =S_{r}+S_{f} .
\end{aligned}
$$

Define a frequently appearing static moment term as

$$
S_{u}=m_{f} u+f m_{t} x_{t} .
$$

Now the linearized equations of motion for the bicycle expressed in the degrees of freedom $\mathbf{q}^{d}=(\phi, \delta)^{T}$ have the form

$$
\mathbf{M} \ddot{\mathbf{q}}^{d}+[\mathbf{C} \mathbf{1} \cdot v] \dot{\mathbf{q}}^{d}+\left[\mathbf{K} \mathbf{0}+\mathbf{K} \mathbf{2} \cdot v^{2}\right] \mathbf{q}^{d}=\mathbf{f}^{d},
$$

with a constant mass matrix, M, a "damping" matrix, C1, which is proportional to the forward speed $v$, and a stiffness matrix which has a constant part, K0, and a part, K2, which is proportional to the square of the forward speed. The forces on the right-hand side, $\mathbf{f}^{d}$, are the applied forces which are energetically dual to the degrees of freedom $\mathbf{q}^{d}$. For the bicycle model the first is $M_{\phi}$, the action-reaction roll moment between the fixed space and the rear frame. In practice such a torque could be applied by side wind, or by 'training wheels' 


\section{PREPRINT}

located at the rear wheel hub, or by a parent teaching a child to ride by applying either a pure rolling moment or a lateral force. The second force is $M_{\delta}$, the action-reaction steering moment between the rear frame and the front frame. This is the torque that would be applied by a rider's hands, or a steering spring-damper, or even an electronic controller. In the case of an ordinary uncontrolled bicycle, both of these moments are taken to be zero. The elements of the mass matrix are

$$
\begin{aligned}
& M(1,1)=T_{x x} \\
& M(1,2)=F_{\lambda x}+f T_{x z}, \\
& M(2,1)=M(1,2) \\
& M(2,2)=F_{\lambda \lambda}+2 f F_{\lambda z}+f^{2} T_{z z} .
\end{aligned}
$$

The velocity-independent elements of the stiffness matrix are

$$
\begin{aligned}
& K 0(1,1)=g m_{t} z_{t}, \\
& K 0(1,2)=-g S_{u}, \\
& K 0(2,1)=K 0(1,2), \\
& K 0(2,2)=-g S_{u} \sin (\lambda),
\end{aligned}
$$

and the elements of the stiffness matrix to be multiplied by the square of the forward speed are

$$
\begin{aligned}
& K 2(1,1)=0 \\
& K 2(1,2)=\left(S_{t}-m_{t} z_{t}\right) \cos (\lambda) / w \\
& K 2(2,1)=0 \\
& K 2(2,2)=\left(S_{u}+S_{f} \sin (\lambda)\right) \cos (\lambda) / w .
\end{aligned}
$$

Finally, the "damping" matrix which is to be multiplied by the forward speed is given by

$$
\begin{aligned}
& C 1(1,1)=0, \\
& C 1(1,2)=f S_{t}+S_{f} \cos (\lambda)+T_{x z} \cos (\lambda) / w-f m_{t} z_{t}, \\
& C 1(2,1)=-\left(f S_{t}+S_{f} \cos (\lambda)\right), \\
& C 1(2,2)=F_{\lambda z} \cos (\lambda) / w+f\left(S_{u}+T_{z z} \cos (\lambda) / w\right) .
\end{aligned}
$$

\section{Results}

Substitution of the parameter values from Table 1 results in the following values for the entries in the mass matrix from (24),

$$
\mathbf{M}=\left[\begin{array}{r}
80.81210000000002,2.32343142623549 \\
2.32343142623549,0.30126570934256
\end{array}\right]
$$

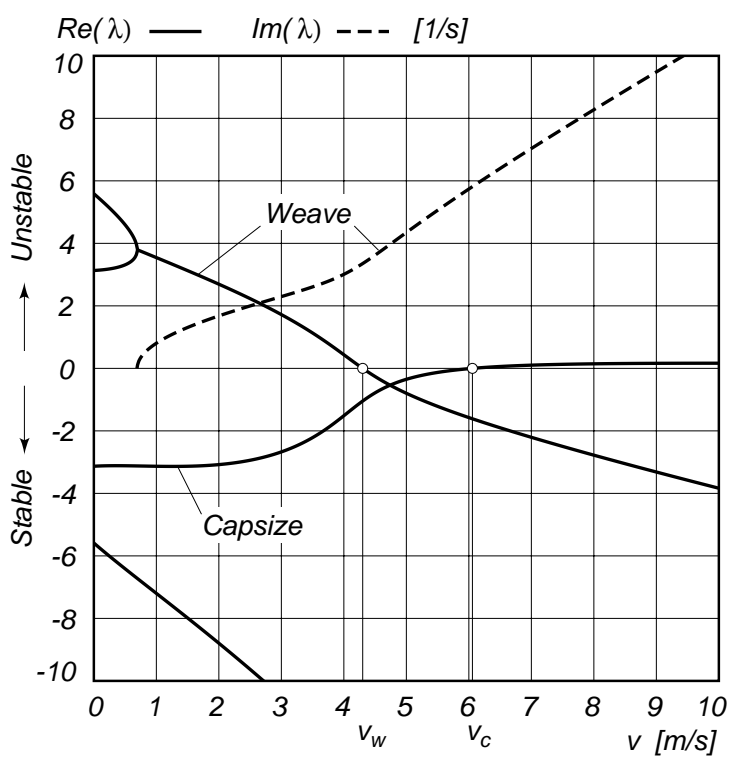

Figure 2. Eigenvalues $\lambda$ from the linearized stability analysis for the benchmark bicycle from Figure 1 and Table 1 where the solid lines correspond to the real part of the eigenvalues and the dashed line corresponds to the imaginary part of the eigenvalues, in the forward speed range of $0 \leq v \leq 10 \mathrm{~m} / \mathrm{s}$. The zero crossings of the real part of the eigenvalues are for the weave motion at at $v_{w}=4.301611 \mathrm{~m} / \mathrm{s}$ and for the capsize motion at $v_{c}=6.057011 \mathrm{~m} / \mathrm{s}$, giving the bicycle an asymptotically stable speed range of $v_{w}<v<v_{c}$

the constant stiffness matrix from (25),

$$
\mathbf{K 0}=\left[\begin{array}{rr}
-794.119500000000, & -25.739089291258 \\
-25.739089291258, & -8.139414705882
\end{array}\right],
$$

the stiffness matrix from (26) which is proportional to the square of the forward speed

$$
\mathbf{K 2}=\left[\begin{array}{lr}
0, & 76.40620875965657 \\
0, & 2.67560553633218
\end{array}\right]
$$

and finally the the "damping" matrix from (27) which depends linearly on the forward speed

$$
\mathbf{C 1}=\left[\begin{array}{rr}
0, & 33.77386947593010 \\
-0.84823447825693, & 1.70696539792387
\end{array}\right] .
$$

\subsection{Stability of the motion}

The stability of the bicycle in the upright steady motion at constant forward speed can be investigated by the homogeneous linearized equations of motion from (23). We start with the usual assumption of an exponential motion with respect to time for the small variations in the degrees of free$\operatorname{dom} \mathbf{q}^{d}=(\phi, \delta)^{T}$ which then takes the form $\mathbf{q}^{d}=\mathbf{q}_{0}^{d} \exp (\lambda t)$. 


\section{PREPRINT}

Substitution into the linearized equations of motion leads to an eigenvalue problem. For the bicycle model under study the characteristic equation of this eigenvalue problem is a polynomial in the eigenvalues $\lambda$ of order four. The coefficients in this polynomial are themselves polynomials in the forward speed $v$, since some coefficients of the linearized equations of motion have a linear or quadratic dependency on $v$. The solutions of the characteristic polynomial for a range of forward speeds are the root loci of the eigenvalues $\lambda$, which are shown in Figure 2. Eigenvalues with a positive real part correspond to unstable motions whereas eigenvalues with a negative real part result in asymptotically stable motions. Complex conjugated eigenvalues give rise to oscillatory motions. For the bicycle model there are two significant eigenmodes, called capsize mode and weave mode. The capsize motion is a non-oscillatory motion in which, when unstable, the bicycle just falls over like a capsizing ship. The weave motion is an oscillatory motion in which the bicycle sways about the headed direction. At very low speed, $0<v<0.5 \mathrm{~m} / \mathrm{s}$, there are two positive and two negative eigenvalues which correspond to an inverted pendulum-like motion of the bicycle. Around $v=0.6 \mathrm{~m} / \mathrm{s}$ two real eigenvalues become identical and start forming a conjugated pair; this is where the oscillatory weave motion emerges. At first this motion is unstable but at $v_{w}=4.301611 \mathrm{~m} / \mathrm{s}$ these eigenvalues cross the real axis in a Hopf bifurcation and the weave motion becomes stable until infinity. After this bifurcation the frequency of the weave motion is almost proportional to the forward speed. Meanwhile the capsize motion, which was stable for low speed, crosses the real axis in a pitchfork bifurcation at $v_{c}=6.057011 \mathrm{~m} / \mathrm{s}$ and the motion becomes mildly unstable. We call a motion mildly unstable when the eigenvalues have a absolute value which is smaller than $2 \mathrm{~s}^{-1}$, in which case it is fairly easy to stabilize the motion manually. With further increase in speed, the capsize eigenvalue approaches zero. We conclude that the speed range for which the bicycle shows asymptotically stable behaviour is $v_{w}<v<v_{c}$, although from a practical point of view one could say that the bicycle is easy to balance for all speeds above $2 \mathrm{~m} / \mathrm{s}$.

\section{Equations of Motion Derived with the Numeric Program SPACAR}

SPACAR is a program system written in Fortran-77 for dynamic analysis of multibody systems, based on a finite element technique. Starting from the principles as laid out by Besseling [1], this software was initiated in the seventies by Van Der Werff [25], and has been further developed among others by Jonker [6; 7], Meijaard [11], and Schwab [19]. The SPACAR program can handle mechanical systems of rigid and flexible bodies that are interconnected by complex joints in both open and closed kinematic loops and having rolling contacts. The dynamical equations are given for a set of minimal coordinates rather than with the aid of Lagrangian multi- pliers. Besides doing forward dynamic analysis, the system is also capable of deriving the numeric coefficients for the linearized equations of motion in any given configuration and state of motion of the system. With the help of a rather limited number of finite element types it is possible to model a wide class of systems. Typical types of elements are beam, truss and hinge elements, while more specialized elements can be used to model complex joint connections, transmissions of motion [20], and rolling contact as in road vehicles and track-guided vehicles [18;21].

The SPACAR model for the benchmark bicycle is sketched in Figure 4, whereas the input file for the SPACAR program describing this model is presented in Appendix A. The model consists of two knife-edge rigid wheel elements, two rigid bodies for the front and the rear frame, and six hinge elements for describing relative rotations. The elements describing the three degrees of freedom are the relative rotations in: hinge (2) for the roll angle $\phi$, hinge (9) for the steering angle $\delta$, and hinge (4) for the rotation $\theta_{r}$ of the rear wheel with respect to the rear frame. The four kinematic coordinates are described by the $x$ and $y$ components of node $\overrightarrow{9}$ which is the rear-wheel contact point, the relative rotation in hinge (1) for the yaw angle $\psi$, and the relative rotation in hinge (12) for the rotation $\theta_{f}$ of the front wheel with respect to the front frame.

\subsection{Linearized Equations of Motion Derived with the Numeric Program SPACAR}

The resulting matrices of the linearized equations of motion (23) as determined by the numeric program SPACAR are:

$$
\mathbf{M}=\left[\begin{array}{r}
80.81210000000002,2.32343142623549 \\
2.32343142623549,0.30126570934256
\end{array}\right]
$$

$$
\mathbf{K 0}=\left[\begin{array}{rr}
-794.119500000000, & -25.739089291258 \\
-25.739089291258, & -8.139414705882
\end{array}\right],
$$

$$
\mathbf{K} 2=\left[\begin{array}{lr}
0, & 76.40620875965666 \\
0, & 2.67560553633216
\end{array}\right]
$$

$$
\mathbf{C 1}=\left[\begin{array}{rr}
0.00000000000005, & 33.77386947593010 \\
-0.84823447825693, & 1.70696539792387
\end{array}\right] \text {. }
$$

These agree with the values given in Section 4, where at most the fifteenth digit may differ a unit. 


\section{PREPRINT}

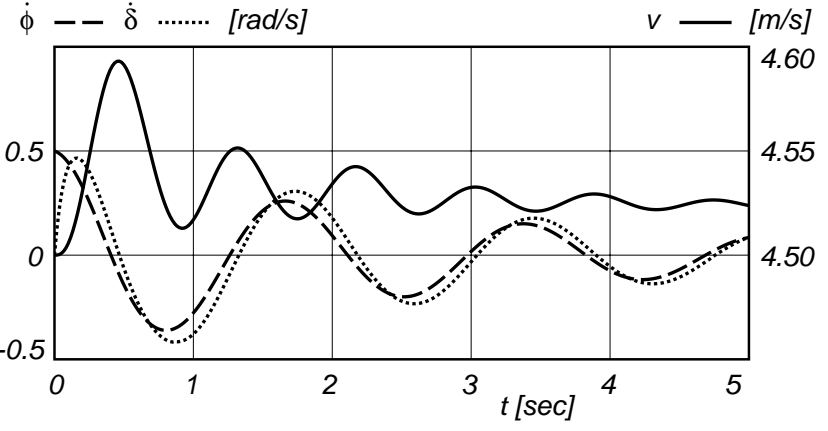

Figure 3. Non-linear dynamic response of the benchmark bicycle from Figure 1 and Table 1 , with the angular roll velocity $\dot{\phi}$, the angular steering velocity $\dot{\delta}$, and the forward speed $v=-\dot{\theta}_{r} R_{r w}$ for the initial conditions: $\left(\phi, \delta, \theta_{r}\right)_{0}=(0,0,0)$ and $(\dot{\phi}, \dot{\delta}, v)_{0}=$ $(0.5 \mathrm{rad} / \mathrm{s}, 0,4.5 \mathrm{~m} / \mathrm{s})$ for a time period of 5 seconds.

\subsection{Non-linear dynamic response}

When an uncontrolled bicycle is in its stable speed range, roll and steer perturbations die away, in a seemingly damped fashion. However, the entire system is energy conservative, and what has happened is that the perturbation energy has been transferred into energy of forward travel. As the forward speed is affected only to second order, linearized equations do not capture this. Therefore a non-linear dynamic analysis with SPACAR is performed on the benchmark bicycle model to demonstrate this phenomenon. The initial conditions at $t=0$ are an upright configuration, $\left(\phi, \delta, \theta_{r}\right)=(0,0,0)$, at a forward speed of $v=4.5 \mathrm{~m} / \mathrm{s}$, which is within the stable speed range of the linearized analysis, and a small perturbation of the angular roll velocity of $\dot{\phi}=0.5 \mathrm{rad} / \mathrm{s}$. Then, in Figure 3, the dynamic response clearly shows a small increase of the forward velocity $v$ while the perturbed lateral motions die out. In the same figure we see that the period for the roll and the steer motion is approximately $T_{0}=1.73 \mathrm{~s}$, which compares well with the $1.734475 \mathrm{~s}$ from the linearized stability analysis. Note also the small phase lag of the steering motion $\dot{\delta}$ relative to the roll motion $\dot{\phi}$.

\section{Linearized Equations of Motion Derived with the Symbolic Program Autosim}

With the multibody dynamics program AutoSim [16; 17], the equations of motion for a mechanical system can be derived in a symbolic form. The program is mainly designed for analysing systems of rigid bodies that are interconnected by prismatic and revolute joints and are arranged in a tree topology. Additional constraints can be imposed on the system for taking into account kinematic closed loops, special joints or non-holonomic constraints. Additional holonomic constraints, however, cannot be solved in general in a symbolic form for the dependent coordinates: an iterative numerical solution for these coordinates is needed, which destroys the purely symbolic nature of the equations. Non-holonomic constraints are generally linear in the velocities and can be solved for the dependent velocities.

The methods used for deriving the equations of motion are mainly based on Kane's approach [8], with some minor modifications. The program is written in Lisp [23] and consists of a set of definitions of functions, macros and data structures. The definitions give procedures for handling algebraic expressions, for modelling of components of multibody systems such as bodies, points, joints and forces, for formulating the equations of motion and for generating output. The input file for an analysis is a Lisp program and the full language is available to the user. The modeller has a fairly good control over the formulation of the equations of motion, while user-defined forces are easy to add.

The equations of motion are obtained in the form

$$
\begin{aligned}
& \dot{\mathbf{q}}=\mathbf{S}(\mathbf{q}, t) \mathbf{u}, \\
& \dot{\mathbf{u}}=[\mathbf{M}(\mathbf{q}, t)]^{-1} \mathbf{Q}(\mathbf{q}, \mathbf{u}, t) .
\end{aligned}
$$

Here, $\mathbf{q}$ are the generalized coordinates, $\mathbf{u}$ are the generalized velocities, $\mathbf{S}$ is the kinematic matrix that relates the rates of the generalized coordinates to the generalized speeds, $\mathbf{M}$ is the system matrix, and $\mathbf{Q}$ contains all force terms and velocity dependent inertia terms.

A standard option for linearization is available, which, however, is not applicable for systems with closed kinematic loops (e.g. the front-wheel ground contact of a bicycle). Fortunately, for the highly symmetric bicycle model, the dependent coordinate, the pitch angle, remains zero to first order, for which special case the linearization option gives the right results. The output consists of a MatLab script file that calculates the matrices of the linearized equations.

The input file used for the bicycle model is listed in Appendix B. The generalized coordinates and velocities are the same as those in the SPACAR model. Two massless intermediate reference frames have been introduced: the yawing frame describes the in-plane translation and yawing of the rear frame, and the rolling frame describes the rolling of the rear frame with respect to the yawing frame. These additional frames automatically satisfy the holonomic constraint at the rear wheel, and also give a better control over the choice of the generalized coordinates. The holonomic constraint at the front wheel and the four non-holonomic constraints are explicitly defined in the input file.

The resulting matrices of the linearized equations of motion (23) are:

$$
\mathbf{M}=\left[\begin{array}{r}
80.81210000000004,2.32343142623549 \\
2.32343142623549, \\
0.30126570934256
\end{array}\right],
$$




\section{PREPRINT}

$$
\begin{gathered}
\mathbf{K 0}=\left[\begin{array}{rr}
-794.199500000000, & -25.739089291258 \\
-25.739089291258, & -8.139414705882
\end{array}\right], \\
\mathbf{K 2}=\left[\begin{array}{rr}
0, & 76.40620875965669 \\
0, & 2.67560553633217
\end{array}\right], \\
\mathbf{C 1}=\left[\begin{array}{rr}
0,33.77386947593011 \\
-0.84823447825693, & 1.70696539792387
\end{array}\right] .
\end{gathered}
$$

These agree with the values given in Section 4, where at most the fifteenth digit may differ a unit.

\section{Conclusions}

If we compare the results obtained by the three methods, it appears that the coefficients for the linearized equations agree with each other and the difference are only caused by the finite precision of the numeric calculations: the relative errors are less than 1 part in $10^{14}$. This gives us confidence that the presented results are correct and the problem can be used as a benchmark test for multibody dynamics simulations.

Starting from the given basic model for the bicycle, more elaborate models can be developed. These may include the finite width of the tyres, control torque at the handle bar, relative motion between the rider and the rear frame and tyre models that include wheel slips and compliance.

\section{Acknowledgement}

Special thanks are extended to Andy Ruina for stimulating, supporting, hosting, and guiding this work on bicycle dynamics, and to Andrew Dressel for his work on the graphical user interface to the JBike6 program; the Matlab implementation of the here presented linearized equations of motion. The second author, Jaap Meijaard, was supported by the Engineering and Physical Sciences research Council (EPSRC) of the U.K.

\section{A SPACAR Input}

\begin{tabular}{|c|c|c|c|c|c|c|}
\hline hinge & 1 & 2 & 5 & & 0 & 0 \\
\hline nge & 2 & 5 & 6 & & 1 & 0 \\
\hline nge & 3 & 6 & 4 & & 0 & -1 \\
\hline nge & 4 & 4 & 8 & & 0 & -1 \\
\hline eel & 5 & 7 & 8 & 9 & 0 & -1 \\
\hline nbody & 6 & 1 & 2 & 9 & & \\
\hline nbody & 7 & 3 & 4 & 7 & & \\
\hline nbody & 8 & 3 & 4 & 10 & & \\
\hline hae & 9 & 4 & 11 & & 1 & 0 \\
\hline hoody & 10 & 10 & 11 & 12 & & \\
\hline
\end{tabular}

The corresponding sketch of the model for generating the SPACAR input file is shown in Figure 4.

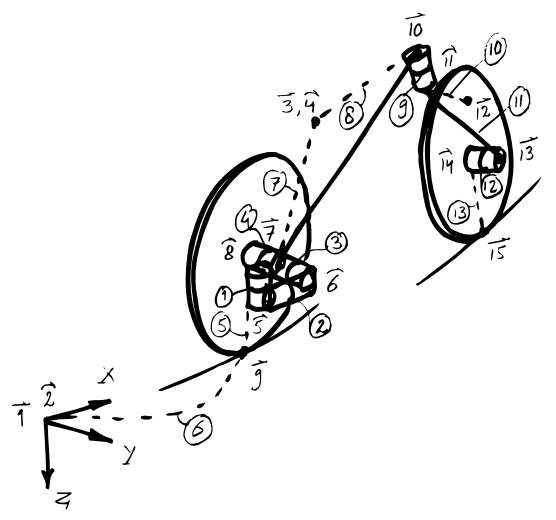

Figure 4. Sketch of the bicycle model for SPACAR input together with node numbers, with straight arrows for positions, curved arrows for orientations, and element numbers encircled.

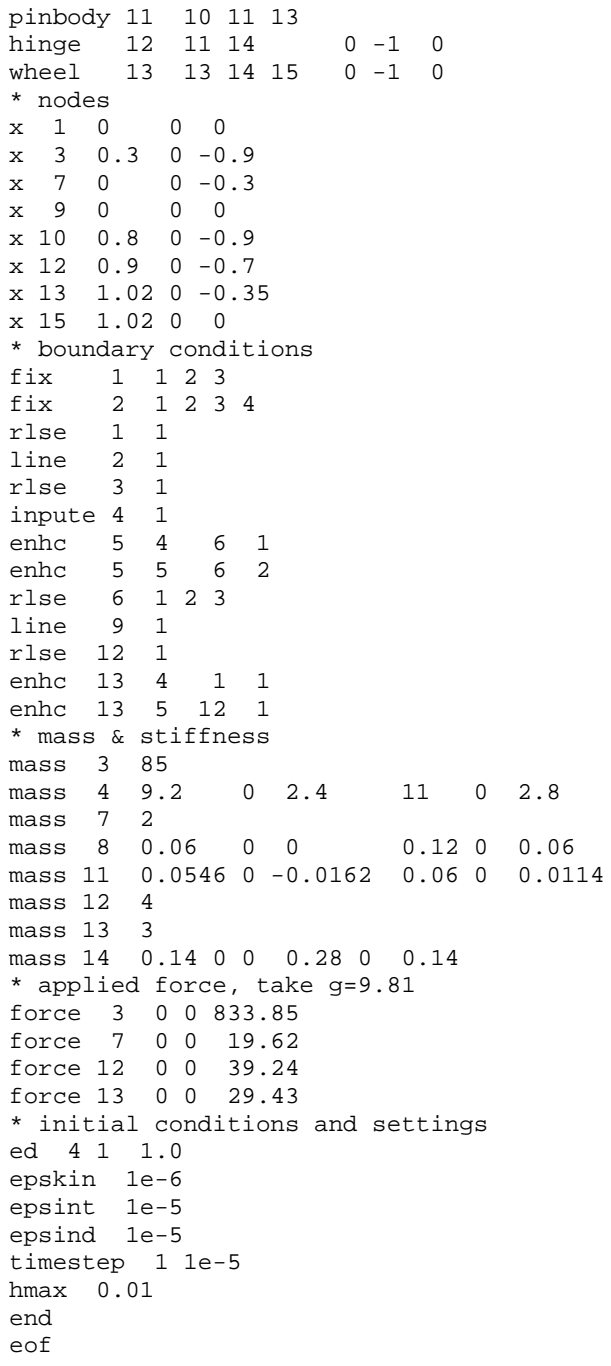




\section{PREPRINT}

\section{B AutoSim Input}

; $;$; This is the file fiets.lsp, with the benchmark1 model. ; ; Set up preliminaries: (reset)

(si)

(add-gravity :direction [nz] : gees g)

(set-names $g$ "Acceleration of gravity" )

(set-defaults $\mathrm{g}$ 9.81) ; this value is used in the benchmark. ; The name of the model is set to the string "fiets"

(setsym *multibody-system-name* "fiets")

$\therefore$; Introduce a massless moving reference frame. This frame

; ; has $\mathrm{x}$ and $\mathrm{y}$ translational degrees of freedoms and a yaw

; ; rotational degree of freedom.

( add-body yawframe :name "moving yawing reference frame" :parent $\mathrm{n}$ :translate ( $\mathrm{x}$ y) :body-rotation-axes $\mathrm{z}$ :parent-rotation-axis $\mathrm{z}$ :reference-axis $\mathrm{x}$ :mass 0 :inertia-matrix 0 )

;; Introduce another massless moving reference frame. This

; ; frame has a rolling (rotational about a longitudinal

i; axis) degree of freedom.

( add-body rollframe :name "moving rolling reference frame" :parent yawframe :body-rotation-axes (x) :parent-rotation-axis $\mathrm{x}$ :reference-axis y :mass 0 :inertia-matrix 0 )

; ; Add the rear frame of the bicycle. The rear frame has a

;; pitching (rotation about the local lateral y-axis of the

; ; frame) degree of freedom.

( add-body rear : name "rear frame" : parent rollframe :joint-coordinates ( 0 o "-Rrw") :body-rotation-axes y :parent-rotation-axis y :reference-axis z :cm-coordinates (bb 0 "Rrw-hh") :mass $\mathrm{Mr}$ :inertia-matrix ( ( Irxx 0 Irxz) ( 0 Iryy 0$) \quad\left(\begin{array}{llll}I r x z & 0 & \operatorname{Irzz}))\end{array}\right)$ set-names

Rrw "Rear wheel radius"

bb "Longitudinal distance to the c.o.m. of the rear frame"

hh "Height of the centre of gravity of the rear frame" Mr "Mass of the rear frame"

Irxx "Longitudinal moment of inertia of the rear frame"

Irxz "Minus product of inertia of the rear frame"

Iryy "Transversal moment of inertia of the rear frame"

Irzz "Vertical moment of inertia of the rear frame" )

(set-defaults Rrw 0.30 bb 0.3 hh 0.9

Mr 85.0 Irxx 9.2 Irxz 2.4 Iryy 11.0 Irzz 2.8 )

$\therefore$ Add the rear wheel of the vehicle. This body rotates

; ; about the $y$ axis of its physical parent, the rear frame.

( add-body $r w$ : name "rear wheel" :parent rear

:body-rotation-axes y :parent-rotation-axis y

:reference-axis $z$ : joint-coordinates $\left(\begin{array}{lll}0 & 0 & 0\end{array}\right)$ :mass Mrw :inertia-matrix (irwx "2.0*irwx" irwx) )

( set-names

Mrw "mass of the rear wheel"

irwx "rear wheel in-plane moment of inertia"

(set-defaults Mrw 2.0 irwx 0.06 )

;; Now we proceed with the front frame.

; Define the steering and reference axes of the front frame:

; ; Add in the front frame: define some points

( add-point head : name "steering head point B" :body $\mathrm{n}$ :coordinates (xcohead 0 zcohead) )

( add-point frontcmpoint : name "c.o.m. of the front frame" :body $\mathrm{n}$ : coordinates ( $\mathrm{xfcm} 0 \mathrm{zfcm}$ ) )

( set-names

epsilon "steering head angle"

xcohead " $x$ coordinate of the steering head point $B$ " zcohead " $z$ coordinate of the steering head point $B "$ xfcm "x coordinate of the c.o.m. of the front frame" zfcm "z coordinate of the c.o.m. of the front frame"

set-defaults epsilon 0.321750554396642163

xcohead 0.80 zcohead -0.90 xf cm 0.90 zfcm -0.70 )

( add-body front : name "front frame" :parent rear

:body-rotation-axes $\mathrm{z}$ :parent-rotation-axis

"sin (epsilon) * [rearx]+cos (epsilon) * [rearz] "

:reference-axis "cos (epsilon) *[rearx]-sin(epsilon) *[rearz]"

:joint-coordinates head : $\mathrm{cm}$-coordinates frontcmpoint

:mass Mf

:inertia-matrix ( (Ifxx 0 Ifxz) ( $\left.\begin{array}{llllll}\text { Ify } & 0\end{array}\right)$ (Ifxz 0 Ifzz)

:inertia-matrix-coordinate-system $\mathrm{n}$ )

( set-names

Mf "Mass of the front frame assembly"

Ifxx "Longitudinal moment of inertia of the front frame"

Ifxz "Minus product of inertia of the front frame"
Ifyy "Transversal moment of inertia of the front frame" Ifzz "Vertical moment of inertia of the front frame" )

( set-defaults Mf 4.0

Ifxx 0.0546 Ifxz -0.0162 Ifyy 0.06 Ifzz 0.0114 )

; ; Add in the front wheel:

( add-point fw_centre : name "Front wheel centre point" :body $\mathrm{n}$ :coordinates ( 110 "-Rfw") )

add-body $\mathrm{fw}$ : name "front wheel" : parent front :body-rotation-axes y :parent-rotation-axis y :reference-axis "[nz]" :joint-coordinates fw_centre :mass Mfw :inertia-matrix (ifwx "2.0*ifwx" ifwx) )

( set-names

11 "Wheel base"

Rfw "Radius of the front wheel"

Mfw "Mass of the front wheel"

ifwx "In-plane moment of inertia of the front wheel")

(set-defaults 11 1.02 Rfw $0.35 \mathrm{Mfw} 3.0$ ifwx 0.14)

$\therefore$ The system is now complete,

; ; except for the contact constraints at the wheels.

;; The holonomic constraint at the rear wheel is

; ; automatically satisfied. The rear wheel slip is zero.

( add-speed-constraint

"dot (vel (yawframe0), [yawframex]) +Rrw* (ru (rear) +ru (rw)) " :u "tu (yawframe, 1) " )

( add-speed-constraint "dot(vel (yawframe0), [yawframey]) " : u "tu (yawframe, 2) " )

$\therefore$ For the front wheel we have a holonomic constraint for

$i$ i the contact and two non-holonomic slip constraints.

;; The slip velocities are defined now.

(setsym singammafw "dot ([fwy], [nz]) ")

(setsym cosgammafw "sqrt (1-@singammafw**2) ")

( setsym fw_rad "([nz] - [fwy] *@singammafw)/@cosgammafw")

( setsym slipfw_long

"dot (vel $\left.(\mathrm{fw} 0)+\mathrm{Rfw}^{*} \operatorname{cross}(\operatorname{rot}(\mathrm{fw}), \mathrm{efw} \mathrm{fad}),[\mathrm{nx}]\right)$ ) )

; ; No longitudinal slip on front wheel;

i; eliminate rotational velocity about the axis

(add-speed-constraint "@slipfw_long" : u "ru(fw)")

; ; normal constraint; eliminate the pitch angle

( setsym slipfw_n

"dot (vel (fw0) +Rfw* $\operatorname{cross}(\operatorname{rot}(\mathrm{fw})$, e fw_rad), [nz]) " )

(add-speed-constraint "@slipfw_n" : u "ru(rear)")

( add-position-constraint

"dot (pos (fw0), [nz])+Rfw*ecosgammafw" :q "rq(rear) ")

; ; No lateral slip on front wheel;

; ; eliminate yaw rate of the yawing frame

( setsym slipfw lat

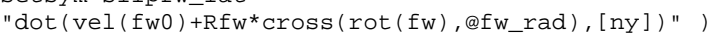

(add-speed-constraint "@slipfw_lat" : u "ru(yawframe)") (dynamics)

(linear)

\section{References}

[1] Besseling, J. F., "The complete analogy between the matrix equations and the continuous field equations of structural analysis," in International symposium on analogue and digital techniques applied to aeronautics: Proceedings, Presses Académiques Européennes, Bruxelles, 1964, pp. 223-242.

[2] Carvallo, M. E., "Théorie du mouvement du Monocycle et de la Bicyclette," Journal de L'Ecole Polytechnique, Series 2, Part 1, Volume 5, "Cerceau et Monocyle", 1900, pp. 119-188, Part 2, Volume 6, "Théorie de la Bicyclette", 1901, pp. 1-118.

[3] Den Hartog, J. P., Mechanics, McGraw-Hill, New York and London, 1948.

[4] Döhring, E., "Stability of single-track vehicles," (Translated by J. Lotsof, March 1957) Technical report, Institut für Fahrzeugtechnik, Technische Hochschule Braunschweig, Forschung Ing.-Wes. 21(2), 1955, pp 50-62. 
[5] Hand, R. S., Comparisons and Stability Analysis of Linearized Equations of Motion for a Basic Bicycle Model, MSc Thesis, Cornell University, May 1988.

[6] Jonker, J. B., A Finite Element Dynamic Analysis of Flexible Spatial Mechanisms and Manipulators, PhD Thesis, Delft University Press, Delft, 1988.

[7] Jonker, J. B., and Meijaard, J. P., "SPACAR-Computer program for dynamic analysis of flexible spatial mechanisms and manipulators," in Multibody Systems Handbook, W. Schiehlen (ed.), Springer-Verlag, Berlin, 1990, pp. 123-143.

[8] Kane, T.R., Dynamics, Holt, Rinehart and Winston, New York, 1968.

[9] Kane, T. R., "Fundamental kinematic relationships for single-track vehicles", International Journal for Mechanical Sciences 17, 1975, pp. 499-504.

[10] Klein, F., and Sommerfeld, A., Über die Theorie des Kreisels, (Chapter IX, Section 8, "Stabilität des Fahrrads", 1910, pp. 863-884.), Teubner, Leipzig, 1897.

[11] Meijaard, J. P., "Direct determination of periodic solutions of the dynamical equations of flexible mechanisms and manipulators," International Journal for Numerical Methods in Engineering 32, 1991, pp. 1691-1710.

[12] Neĭmark, Ju. I., and Fufaev, N. A., Dynamics of Nonholonomic Systems, (Translated from the Russian edition, Nauka, Moscow, 1967), A.M.S., Providence RI, 1972.

[13] Papadopoulos, J. M., "Bicycle Steering Dynamics and Self-Stability: a Summary Report on Work in Progress", Technical report, Cornell Bicycle Research Project, 1987, pp. 1-23 (availabe at: http://tam.cornell.edu/ruina/hplab/bicycles.html).

[14] Rankine, W. J. M., "On the Dynamical Principles of the Motion of Velocipedes," The Engineer 28, 1869, pp. 79, 129, 153, 175.

[15] Society of Automotive Engineers, "Vehicle Dynamics Terminology-SAE J670e," 2001 SAE Handbook, SAE international, Warrendale, PA, 2001.

[16] Sayers, M.W., "Symbolic Computer Language for Multibody Systems," Journal of Guidance, Control, and Dynamics 14, 1991, pp. 1153-1163.

[17] Sayers, M.W., "Symbolic Vector/Dyadic Multibody Formalism for Tree-Topology Systems," Journal of Guidance, Control, and Dynamics 14, 1991, pp. 12401250.

[18] Schwab, A. L., and Meijaard, J. P., "Dynamics of Flexible Multibody Systems with Non-Holonomic Constraints: A Finite Element Approach," Multibody System Dynamics 10, 2003, pp. 107-123.

[19] Schwab, A. L., Dynamics of Flexible Multibody Systems, PhD Thesis, Delft University of Technology, Delft, April 2002.
[20] Schwab, A. L., and Meijaard, J. P., "The belt, gear, bearing and hinge as special finite elements for kinematic and dynamic analysis of mechanisms and machines," in Leinonen, T. (ed.), Proceedings of the Tenth World Congress on the Theory of Machines and Mechanisms, IFToMM, June 20-24, 1999, Oulu, Finland, Oulu University Press, Vol 4, pp. 1375-1386.

[21] Schwab, A. L., and Meijaard, J. P., "Dynamics of flexible multibody systems having rolling contact: Application of the wheel element to the dynamics of road vehicles," Vehicle System Dynamics Supplement 33 (1999), pp. 338-349.

[22] Sharp, R. S. , "The Stability and Control of Motorcycles," Journal of Mechanical Engineering Science, 13(5), 1971, pp. 316-329.

[23] Steele, G.L., Jr, 1990. Common Lisp, The Language, 2nd ed., Digital Press, U.S.A., 1990.

[24] Timoshenko, S., and Young, D. H., Advanced Dynamics, McGraw-Hill Book Company, New York, 1948.

[25] Van Der Werff, K., Kinematic and Dynamic Analysis of Mechanisms, a Finite Element Approach, PhD Thesis, Delft University Press, Delft, 1977.

[26] Weir, D. H., Motorcycle Handling Dynamics and Rider Control and the Effect of Design Configuration on Response and Performance, $\mathrm{PhD}$ Thesis, University of California, LA, 1972.

[27] Weir, D. H., and Zellner, J. W., "Lateral-directional motorcycle dynamics and rider control," Technical report 780304, Society of Automotive Engineers, Warrendale, PA, 1978.

[28] Whipple, F. J. W., "The Stability of the Motion of a Bicycle," The Quarterly Journal of Pure and Applied Mathematics 30, 1899, pp. 312-348. 